\title{
Crack detection in oak flooring lamellae using ultrasound-excited thermography
}

\author{
Tobias Pahlberg ${ }^{\mathrm{a}, *}$, Matthew Thurley $^{\mathrm{b}}$, Djordje Popovic ${ }^{\mathrm{c}}$, Olle Hagman ${ }^{\mathrm{a}}$ \\ ${ }^{a}$ Luleå University of Technology, Campus Skellefteå, \\ Forskargatan 1, SE-931 87 Skellefteå, Sweden \\ ${ }^{b}$ Luleå University of Technology, \\ SE-971 87 Luleå, Sweden \\ c Jönköping University, Gjuterigatan 5, SE-551 11, Jönköping, Sweden
}

\begin{abstract}
Today, a large number of people are manually grading and detecting defects in wooden lamellae in the parquet flooring industry. This paper investigates the possibility of using the ensemble methods random forests and boosting to automatically detect cracks using ultrasound-excited thermography and a variety of predictor variables. When friction occurs in thin cracks, they become warm and thus visible to a thermographic camera. Several image processing techniques have been used to suppress the noise and enhance probable cracks in the images. The most successful predictor variables captured the upper part of the heat distribution, such as the maximum temperature, kurtosis and percentile values $92-100$ of the edge pixels. The texture in the images was captured by Completed Local Binary Pattern histograms and cracks were also segmented by background suppression and thresholding.

The classification accuracy was significantly improved from previous research through added image processing, introduction of more predictors, and by using automated machine learning. The best ensemble methods reach an average classification accuracy of 0.8 , which is very close to the authors' own manual attempt at separating the images (0.83).

Keywords: Ultrasound-excited thermography, Crack detection, Parquet flooring, Wood, Ensemble classification, Machine learning
\end{abstract}


5 2020, with China as the fastest growing mar-

6 ket (Global Industry Analysts, Inc., 2017). In

7 2013, engineered wood flooring accounted for s $50 \%$ of the total wood flooring sales (Info9 graphic Journal, 2013).

Tarkett is one of many producers of floorboards from engineered wood. In general, their floorboards are made out of three layers, where the top layer consists of 3-4 mm thick lamellae made of some expensive hardwood species. This particular raw material starts out as laths that are partly dried naturally in a storage compartment. The laths are dried in kilns for a certain period of time depending on the moisture content just before the processing is about to begin. The dried laths are planed, cross-cut, and rip-sawn in the factory in order to produce lamellae of the desired shape

The lamellae are then manually inspected and

${ }^{*}$ Corresponding author. Address: Luleå University 48 of Technology, Campus Skellefteå, Forskargatan 1, SE93187 Skellefteå, Sweden. Telephone: +46 (0)910585336

Email address: tobias.pahlberg@ltu.se (Tobias Pahlberg) sorted according to different wood features, such as color and the presence of knots and cracks.

There are two types of cracks in the floorboard production: acceptable and unacceptable. Acceptable cracks are very thin and short both in depth and length. Unacceptable cracks are those that are large and have an angle close to horizontal against the lamella face. Moreover, cracks are generally not accepted very close to the lamellae edges. The majority of the cracks are detected on the cross-section of the lamellae, but may also be found on the faces and/or the sides.

Thin and short cracks are often difficult to identify with manual inspection. Lamellae having fine vertical cracks do not pose a problem in the final product since these cracks will be filled and then sealed by lacquer. The biggest problem is when an unacceptable crack is not noticed and this lamella reaches further processing.

Three surface quality inspections are currently used to detect defective floorboards: right after the lacquering line, at the packaging line, and at the finished goods audit. These inspections engage quite a lot of resources, and there is also a cost for the reworking of all the floorboards having surface defects. Tar- 


\subsection{Cracks and crack detection in wood}

kett has estimated possible savings of 100,000 79

Euros per year for the production of 2 million 80 square meters of floorboard if no reworking 81 was needed.

Cracks in wood can form for several differ- ${ }_{87}$ ent reasons. According to Lamb (1992), they ${ }_{88}$ can be caused by, for example, growth stresses, 89 machining, kiln drying, or they can form at ${ }_{90}$ a later stage from anisotropic shrinking and 91 swelling caused by the surrounding climate. 92 Cracks can also form because of mechanical ${ }_{93}$ damage and faulty design.

When wood is excited by vibrations in the 95 ultrasonic frequency range ( $>20 \mathrm{kHz})$, defects 96 such as cracks and other loose material may 97 get hot depending on the defect and the partic- 98 ular sonic excitation and hence be detectable 99 by a thermographic camera. Thin cracks be- 100 come hot due to the friction that occurs be- 101 tween the surfaces. This phenomena is the ba- 102 sis for the crack detection presented in this pa- 103 per. Open cracks do not generally heat up be- 104 cause there is no friction there. However, open 105 cracks will still heat up at the crack tips (Guo 106 and Vavilov, 2013).

\subsection{Infrared thermography}

Infrared thermography (IRT) has become a widely accepted tool for real-time monitoring and detection of flaws in equipment and detection of defects in materials (Bagavathiappan et al., 2013). As IR cameras are improving and at the same time getting cheaper, more and more applications are becoming feasible.

Thermography methods can provide data about the subsurface structure by observing differences in thermal emission from the object. The emission is the result of a heat transfer through the piece. The thermography is called active if there is an external energy source that raises the temperature of the measured object, initiating the heat transfer. Variants of active thermography methods are: pulse, step heating, lock-in and vibrothermography (Maldague, 2001). Ultrasoundexcited thermography (UET), which is used in this study, is one of the variants of vibrothermography. Unlike most thermography methods, UET is a contact method. A sonotrode is brought into physical contact with the test piece in order to excite the object with a mechanical wave. Ultrasonic excitation generates three-dimensional vibrations that travel through the material (Guo and Vavilov, 2013). The interaction between the waves and the 
composition of the material, together with its 136 shape, give the regions their different tempera- ${ }_{137}$ tures.

When monitoring for faults and defects us- 139 ing thermography, it is common to compare 140 the heat pattern of the defect-free version of 141 the object with that of the faulty one. This procedure is quite trivial on objects made of ${ }^{142}$ homogeneous materials. However, for hetero- ${ }_{143}$ geneous materials such as wood, it is difficult 144 to estimate an accurate reference map prior to 145 the excitation. Often, the boundaries between 146 earlywood and latewood light up in the IR im- 147 age. The annual ring pattern can of course ${ }_{148}$ be observed even without an IR camera but it ${ }_{149}$ is still impossible to know beforehand exactly 150 how much each annual ring will heat up.

\subsection{Research question and aim}

This paper is a continuation of the Mas- ${ }^{154}$ ter's thesis research of Popovic (2015). Ex- 155 periments are performed to further investigate 156 the use of vibrothermography for the detection 157 of cracks in parquet lamellae, and the aim is 158 to improve the accuracy of the classification. 159 The classification accuracy of commonly used 160 ensemble classifier techniques is evaluated for 161 random forests and boosting. The investigated 162 variables are the maximum temperature, the 163 statistical moments of the temperature distribution, the histogram/percentiles, the texture, and classifier-specific parameter settings.

Our hypothesis is that many different types of variable can contribute to a stronger classifier in all.

\subsection{Related work}

Thermography has already been applied in wood science and the wood industry to address different types of problems. Berglind (2003, pp. 67-68) used pulsed heating and lock-in thermography for the purpose of detecting glue deficiency in laminated wood. Lock-in thermography was recommended for its greater penetration depth, up to $3 \mathrm{~mm}$ layer thickness, and for its high signal-to-noise ratio. Sembach and Busse (1997) used lock-in thermography to detect air channels beneath the surface of medium density fiberboards and chipboards. Wu and Busse (1995) used lockin thermography and managed to detect holes with a diameter of $4 \mathrm{~mm}$, knots, and differences in wood species, beneath a $2 \mathrm{~mm}$ layer of laminated veneer. Meinlschmidt (2005) used on-line thermography to detect defects in wood and wood-based materials. Defects such as knots, holes, and cracks could be detected in IR images manually using both passive and 
active thermography. Lukowsky (2008) used 193 ultrasound-excited thermography to success- 194 fully detect black knots, loose material, poorly 195 glued bonds, and rot, in solid wood, oriented 196 strand board (OSB), and particle board. More 197 recently, López et al. (2014) investigated the 198 possibility to use long lasting pulse thermog- 199 raphy (60-600 seconds) to detect bore holes of 200 different diameters at different depths in oak, 201 beech, and Scots pine samples.

IRT has also been used in a wide variety of 203 other fields. Investigations of crack detection 204 using UET have, for example, been carried out 205 both with aluminum and concrete (Guo and 206 Vavilov, 2013; Aggelis et al., 2010). An exten- 207 sive review of the applications of IRT was con- 208 ducted by Bagavathiappan et al. (2013). Ap- 209 plications include: monitoring of civil struc- 210 tures, electrical components, machines and 211 machine components, welded joints, deforma- 212 tion of objects, and detection of moisture in 213 different materials. A common denominator is 214 that most of the conducted studies within IRT only include very simple measures to detect ${ }_{215}$ anomalies, such as, for example, the peak- or average temperature.

The paper from now on is organized as fol- 217 lows. Section 2 first gives an introduction 218 to the texture descriptor Local Binary Pat- 219 terns. Then, the chosen classification methods and some related fundamentals are introduced. Lastly, optimization of the classifier settings is touched upon. In Section 3, the properties of the wood are explained and also how the image data was split into different groups. Section 4 shows the experimental setup and lists the equipment used in the experiment. In Section 5, the different types of image noise are explained in detail and the strategies chosen to mitigate them. In Section 6 the chosen predictor variables are explained as well as the related settings that were found optimal. In Section 7 , the choice of classifiers is motivated. We explain how their performance was measured and how their internal parameters were optimized. In Section 8, the general findings and settings are presented for each type of variable. The performance of the ensembles are explained and discussed. Lastly there follow some concluding remarks in Section 9 and suggested future work in Section 10.

\section{Theory}

\subsection{Local Binary Pattern histograms}

Local Binary Pattern (LBP) histograms can be important predictors of crack presence as shall be seen later. A local binary pattern is 

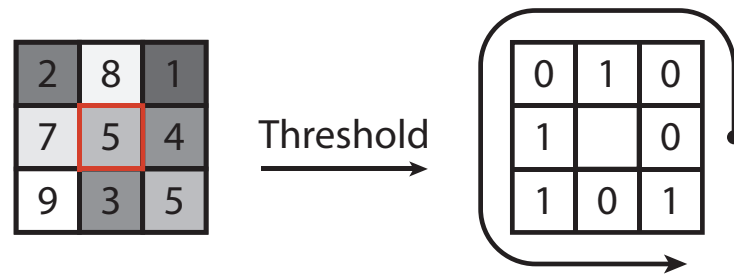

Binary pattern: 00101101

Decimal: $1+4+8+32=45$
Figure 1: If a surrounding value is lower than the center value, it is denoted by a zero, otherwise, by a one. This is a thresholding into a binary pattern, which also is equal to a certain decimal number. a low-level texture operator that can be used 249 to describe the texture at every point in an im- 250 age. The descriptor is obtained by comparing 251 each pixel to its surrounding neighbors. The 252 type of texture is then stored as a binary num- 253 ber, i.e., a sequence of zeros and ones. If $a_{254}$ surrounding value, called the sample point, is 255 lower than the center value, a " 0 " is added to 256 the sequence, otherwise a " 1 " (see an example ${ }_{257}$ in Figure 1). Meaningful micro-features that ${ }_{258}$ are encoded using LBPs are: edges, corners, 259 spots, and smooth surfaces (Figure 2).

All similar local texture features can be 261 summed up into the bins of a histogram which 262 then describes the whole, or parts of the whole 263 image. Thus, each bin provides the probability 264 of encountering the corresponding pattern in 265 the image. The histogram is like a fingerprint 266 of the image, which can be used for recogni- 267 tion and classification.

Binary patterns with zero or two transitions from 0 to 1, and vice versa, are called uniform patterns. This sub-group accounts for about $90 \%$ of all patterns, both in face recognition and in texture recognition, when eight sample points and a radius of one pixel is used (Ahonen et al., 2006; Ojala et al., 2002). According to Ojala et al. (2002), the proportion of nonuniform patterns is so small that their probabilities cannot be estimated reliably. The inclusion of their noisy estimates would actually only impair the performance.

An example of how some binary strings are sorted and put into different bins can be seen in Table 1. The bin number corresponds to the number of consecutive 1's in the circular pattern. Non-uniform patterns, those with more than two 0-1 transitions, are all put in a separate bin. In this example, the pattern rotation is neglected, which in this case, of eight sample points, reduces the number of bins from 59 to 10.

Previous studies using LBP have included, for example, image segmentation (Ojala and Pietikinen, 1999), texture classification (Ojala et al., 2002), face detection (Zhang and Zhao, 2004), and facial expression recognition (Zhao and Pietikainen, 2007). 


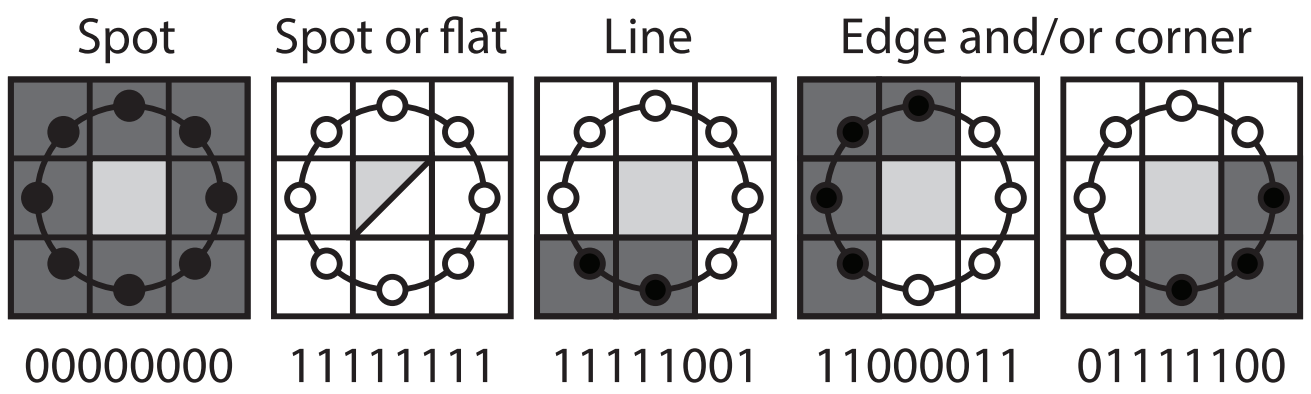

Figure 2: Examples of different uniform LBPs with 8 sample points. Illustration inspired by Wagner (2011).

\subsection{Decision trees}

The classification methods described in this paper all use decision trees in some way. A decision tree is a tree-like graph that can be used to model the behavior of some process. It is a conceptually simple but powerful technique 294 that can either be used for regression or classi- 295 fication. Hence, they are known as regression 296 trees or classification trees, respectively.

The topmost node in a tree is called the root ${ }_{298}$ node. Below the root node there follow a num- 299 ber of internal nodes, and lastly there are the 300 terminal nodes, also called leaves. The further ${ }_{301}$ down the tree, the less important the variables 302 become for class discrimination. An example ${ }_{303}$ of a classification tree which has been learned 304 on the cracked lamellae data can be seen in 305 Figure 3.

Decision trees are usually made as binary 307 trees, meaning that the split at each node is a 308 two-choice question. The reason is that multi- 309 way splits fragment the data too quickly, leaving insufficient data at the next level down (Hastie et al., 2009, p. 311). Multiway splits can still be achieved through a series of binary splits.

\subsection{Ensemble methods}

Ensemble methods are often a good choice of classifier when there is no simple relation between the variables and the output classes. These classifiers are typically made up of several decision trees, and the result is obtained through a vote by all its members. Ensemble voting can be likened to asking a group of people their opinion on a given topic, and then choosing the most popular response. The collected result of many insecure voters will give a quite good guess if they are independent of each other and they are slightly better than random guessing. Some of the most influential techniques that often use decision trees are boosting, bootstrap aggregation, and random 


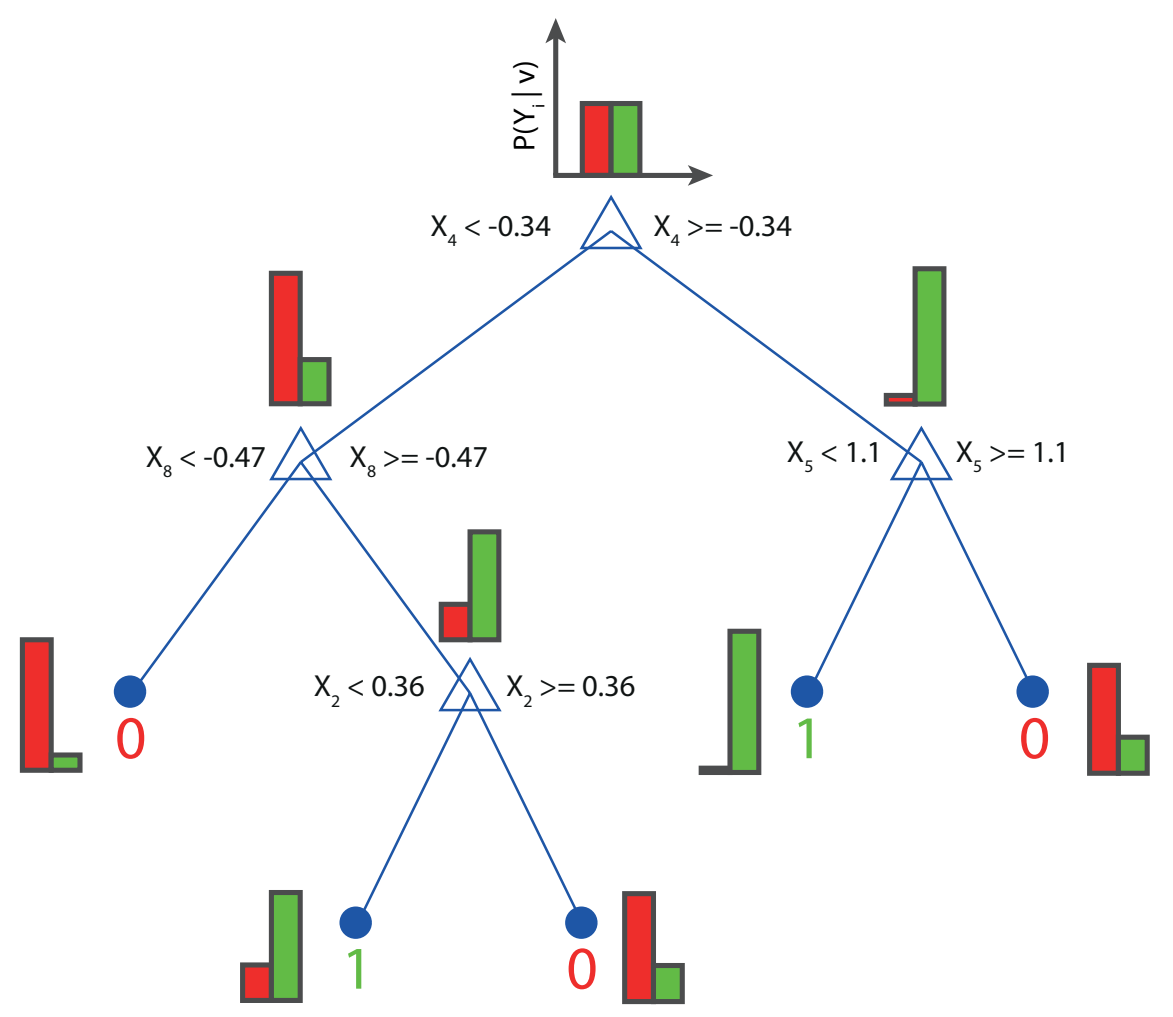

Figure 3: A classification tree learned on the cracked lamellae data. Here, the most discriminating variable on top is the standardized kurtosis of the temperature distribution $\left(X_{4}\right)$. Kurtosis describes the shape of the tails of a distribution. The variable thresholds are shown at each node. The leaf nodes specify the classes where there is a crack present, 1 , or not, 0 . The bars show the probability distribution, $P$, of lamellae at each node belonging to class $Y_{i}$ without cracks (red) and with cracks (green), given the input vector $v$. (Figure best viewed in color)

forests. They are summarized below.

\subsubsection{Boosting}

Boosting is a machine learning technique that creates a strong classifier from the com- ${ }^{323}$ bination of many weak classifiers, or rules ${ }^{324}$ of thumb (Schapire, 2013). The theory of ${ }^{325}$ boosting lies in the weak learning condition ${ }^{326}$ (WLC). The WLC states that the weak classi- ${ }^{327}$ fiers must at least be better than random guess- 328 enough data, the generalization error can become arbitrarily small (Freund and Schapire, 1997; Schapire, 2013).

AdaBoost, short for "adaptive boosting", 
Table 1: An example of the first decimal numbers, bi- ${ }^{340}$ nary strings, and bins they are sorted into. The bin num- 341 ber corresponds to the number of consecutive 1's in the ${ }_{342}$ circular pattern. Non-uniform patterns, those with more than two 0-1 transitions, are all put in the last bin (labeled no. 9 here).

\begin{tabular}{|c|c|c|}
\hline Dec. number & Binary no. & Bin number \\
\hline 0 & 00000000 & 0 \\
\hline 1 & 00000001 & 1 \\
\hline 2 & 00000010 & 1 \\
\hline 3 & 00000011 & 2 \\
\hline 4 & 00000100 & 1 \\
\hline 5 & 00000101 & 9 \\
\hline 6 & 00000110 & 2 \\
\hline 7 & 00000111 & 3 \\
\hline$\vdots$ & $\vdots$ & $\vdots$ \\
\hline 255 & 11111111 & 8 \\
\hline
\end{tabular}

was formulated by Freund and Schapire 357 (1997) and has been extensively studied. It 358 remains one of the most popular and high- 359 performing classifiers two decades after its in- 360 troduction (Schapire, 2013). AdaBoost calls 361 a given weak learning algorithm repeatedly in 362 a series of rounds. The method is adaptive 363 in the sense that subsequently acquired weak 364 classifiers are forced to focus on those obser- 365 vations misclassified by previous classifiers. 366 Each weak classifier produces a hypothesis, 367

$h\left(x_{i}\right)$, for each sample in the training set (Freund and Schapire, 1999). At each iteration, a weak classifier is selected and assigned a coefficient $\alpha_{t}$ such that the sum of the training errors of the resulting boost classifier is minimized.

There have arisen many variants of boosting since the original AdaBoost, for example, LogitBoost and GentleBoost, both introduced by Friedman et al. (1998).

\subsubsection{Bootstrap aggregation and random} forests

Bootstrap aggregation, or bagging, is a technique for reducing the variance of a prediction function (Hastie et al., 2009). Bootstrapping means that several different training

sets are randomly sampled with replacement from the training data, where replacement means that the observations may be picked several times. Bagging averages the prediction over a collection of bootstrap samples, thereby its variance is reduced.

Bagging is compatible with most classifiers but seem to work especially well for highvariance, low-bias procedures, such as decision trees. When used for classification, a committee of trees each cast a vote for the predicted class, and then the majority vote is ac- 
cepted as the answer.

Bagging algorithms generally construct 397 deep trees, and the construction often con- 398 sumes more time and memory than boosting. 399 However, boosted classifiers might need more 400 ensemble members than bagging. It is not al- 401 ways clear which type of classifier is superior 402 (MathWorks MATLAB).

Random forests (Breiman, 2001) is a mod- 404 ification of bagging that improves the variance reduction by reducing the correlation be- 405 tween trees without increasing the variance too much. The de-correlated trees are created by only letting each of them see parts of the whole data, and using a subset of all variables as candidates at each split in the tree.

\section{4. $k$-fold cross-validation $(C V)$ with stratifi- ${ }^{4}$} cation

Cross-validated classification accuracy is used throughout this paper to evaluate the effects of different design choices. When using $\mathrm{CV}$ to evaluate classifier accuracy, stratification is preferred. Stratification means that the $k$-fold partitions are created with the same class distributions as the whole data set. Without stratification, some test sets might include very few observations from certain classes and hence the estimation of the performance for that fold would be misleading (Tsamardinos et al., 2014).

According to Eriksson et al., p. 374, somewhere between $5-10$ is a good general number of groups for $\mathrm{CV}$. This particular interval is the most common one in the literature to obtain an unbiased prediction of the classification $\operatorname{accuracy}^{1}$ (Breiman et al., 1984; Weiss and Indurkhya, 1994; Shen et al., 2007).

\subsection{Hyperparameter optimization}

Hyperparameter optimization is an essential, but often overlooked, step for obtaining a 8 good classifier. A hyperparameter is an inter409 in a tree-based classifier or the number of neurons in a hidden layer of a neural network. All classification methods have their own hyperparameters. Typically, optimizing the hyperparameters means that you try to minimize the cross-validation loss of a classifier in an outer loop (Thornton et al., 2013; MathWorks MATLAB).

Optimal parameters can be found using ${ }_{20}$ manual search, grid search, random search, or

\footnotetext{
${ }^{1}$ Umetric's SIMCA 14 uses 7-fold CV by default and MATLAB's Classification Learner uses 5-fold CV.
} 
automatic search. There is no obvious alter- 450 native better than the other, it depends on the 451 data set and how well the relevant intervals and 452 step lengths are known. Bergstra and Bengio 453 (2012) state that grid search and manual search are the most widely used methods but claim ${ }^{454}$ that random search is more efficient for hyperparameter optimization. By randomly picking values from the grid of hyperparameters, ${ }_{457}$ one can obtain a similar performance as grid search, but with much fewer trials.

In grid search and random search the next trial configuration is picked blindly (Pham, 2016). However, in automatic hyperparameter tuning, an algorithm tries to make a smart choice of new parameters. The algorithm tries to estimate the black-box function that maps ${ }_{465}$ the hyperparameter settings to the classifica- ${ }_{466}$ tion accuracy. Typically, algorithms try to op- ${ }_{467}$ timize the expected improvement (EI), which is the probability that a new trial will result ${ }_{469}$ in a new best level of accuracy. The al- ${ }_{470}$ gorithms all trade-off exploration versus exploitation, to minimize the number of evaluations of the black-box function. Bayesian op- ${ }^{472}$ timization with Gaussian processes as proba- 473 bility model has often been used in the past 474 (Rasmussen and Williams, 2006; Snoek et al., 475 2012). Training a Gaussian process involves 476 fitting a distribution of smooth functions to the given training data. Also, tree search solvers have proved successful in recent years (Hutter et al., 2011; Bergstra et al., 2013).

\section{Material}

In this section, the properties of the wood are explained and also how the image data was split into different groups.

The samples of lamellae are made of oak (Quercus sp.) with the dimensions $304 \times 67 \times 3$ $\mathrm{mm}(\mathrm{L} / \mathrm{W} / \mathrm{T})$. There are three categories into which the lamellae are classified at the manual sorting station: 1) no cracks present, 2) acceptable cracks present and 3) unacceptable cracks present. Workers at the lamella sorting station picked out 150 lamellae from each of these classes. Lamellae with large open cracks were not included in the sample since these are today easily detected at the manual sorting station, and it would also be trivial to find these types of cracks automatically on regular digital images.

\subsection{Definition of a crack}

Tarkett's definition of acceptable and unacceptable cracks is stated below and is explained with the aid of Figure 4. The acceptable cracks satisfy the following criteria: 
a) The crack width is approximately less 503 than $0.2 \mathrm{~mm}$.

b) It has an angle that makes the horizontal length not longer than $7 \mathrm{~mm}$.

c) It is not longer than $20 \mathrm{~mm}$ in the longi- ${ }^{508}$ tudinal direction.

d) It is found more than $5 \mathrm{~mm}$ from the edge 511 of the lamella.

The cracks are regarded as unacceptable if ${ }_{514}$ any of these four characteristics are exceeded. 515

A close ocular inspection of the infrared im- 516 ages did not show any distinct differences be- 517 tween the acceptable cracks and the unaccept- 518 able cracks. When tested, the two types of 519 cracks could also not be clearly separated us- 520 ing computer-aided classification. Both of the 521 crack types heat up due to friction generated 522 by vibrations. The hot area near the cracks ${ }_{523}$ becomes wider than the actual cracks, so it 524 is not possible to determine the crack width 525 from these images. Furthermore, since a sim- 526 ple setup was chosen, with only one IR camera ${ }_{527}$ looking at the lamella face, there was no pos- 528 sibility of determining the angle of the crack. ${ }_{529}$ Hence, the images from the two crack classes 530 were merged into one group.
3.2. Misclassifications and difficulties with the material

Since the visual inspection is done manually, there is a risk of some of the lamellae being misclassified. All lamellae were afterwards investigated with a microscope and as a result 32 lamellae in the group without cracks were found to actually have one or more cracks. These lamellae images were therefore moved to the group with cracks. Most of these lamellae, all but five, had only acceptable cracks. Unfortunately, it is still very difficult to obtain a totally precise truth data for this kind of complex material and specific crack definitions.

In general, there are more bright/hot pixels in the IR images with cracks than in those without. Often, it is quite easy to manually classify an image that has many cracks, but it can be very difficult to distinguish cracks from other possibly hot regions, e.g., growth rings, mechanical resonance modes, or wood dust. Figure 5 shows how the ranges overlap for one of the best predictors of crack presence, the maximum temperature in the image.

The overlap is not substantial for this particular predictor, but as can be seen, the value for the 3rd quartile in the first group coincides with the value of the 1st quartile in the second 


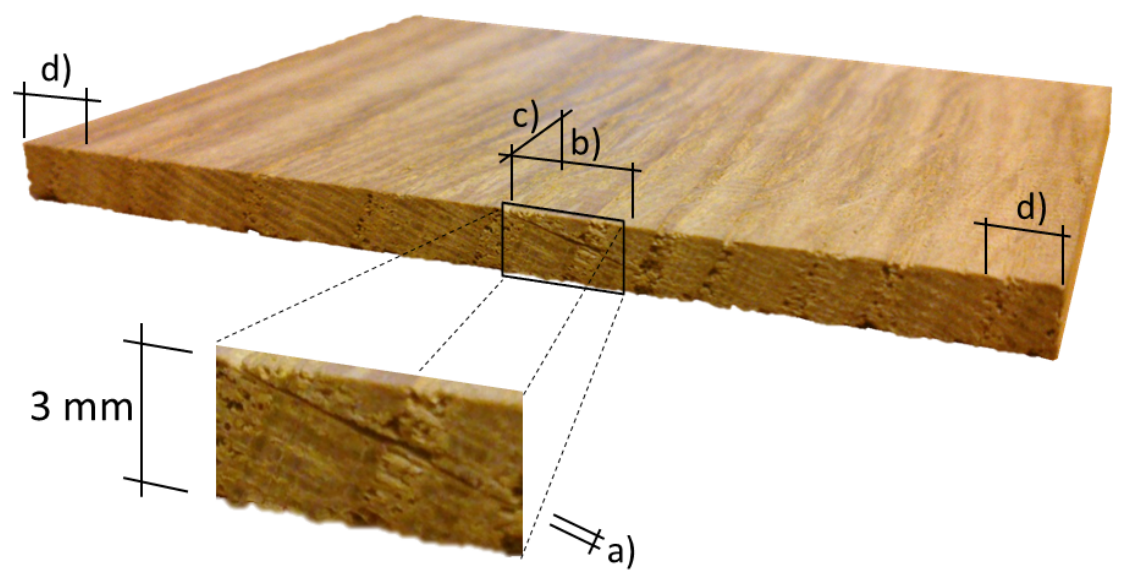

Figure 4: Example of an unacceptable crack that is hard to detect with a quick manual inspection.

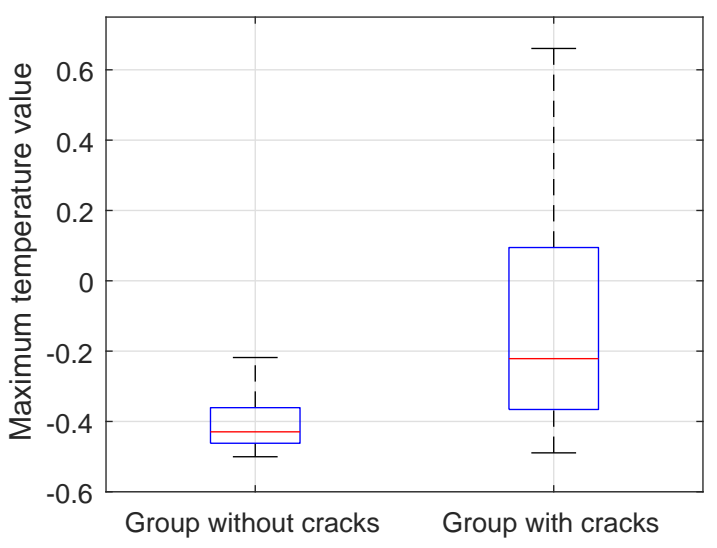

Figure 5: A boxplot showing an example of a predictor with an overlapping range between the group without ${ }^{545}$ cracks and the group with cracks. Standardized maximum temperature value is displayed here. Outliers are not shown here. Whiskers extend to the standard \pm 2.7 standard deviations.

group.

\subsection{Data set imbalance}

After merging the groups that include ${ }_{553}$ cracks, there is a problem with data imbalance, 554 i.e., there is more data with cracks than without. This imbalance will give a classifier biased towards predicting the majority class unless the right precautions are taken. There are several strategies that can be adopted to mitigate this: use methods that handle imbalanced data well, change the performance measure to something other than classification accuracy, or re-sample the data (Brownlee, 2015). The choice was made to re-sample the data.

At this point there were 118 specimens with no cracks and 332 specimens with cracks. A portion of 35 specimens from each of the two classes was randomly picked and put into an 550 sponds to $30 \%$ of the data in the group without cracks. The rest of the data, the training set, 


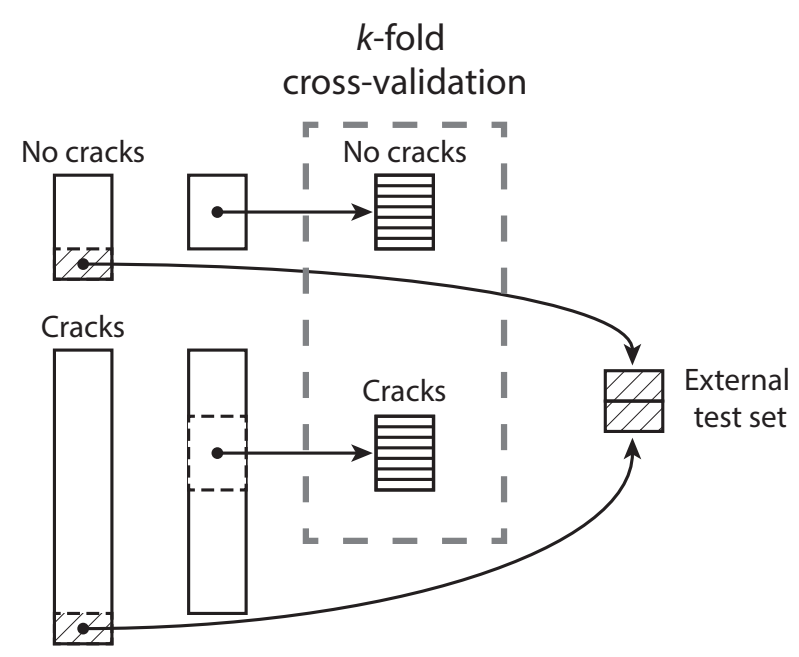

Figure 6: Overview of how the entire data set was divided into a training set for cross-validation and an external test set. rameters.

All specimens without cracks in the training set were always used, but for the group containing cracked lamellae, a random subsample was picked repeatedly, in this way taking care of the problem of the imbalanced data ${ }^{572}$ while still using most of the available data ${ }^{573}$ from the group containing cracks. A schematic ${ }^{574}$ overview of the data divisions is shown in Fig- ${ }^{575}$ ure 6.

\subsection{Raw images}

A video sequence was recorded during the ${ }_{578}$ excitation of each lamella. After analyzing the 579
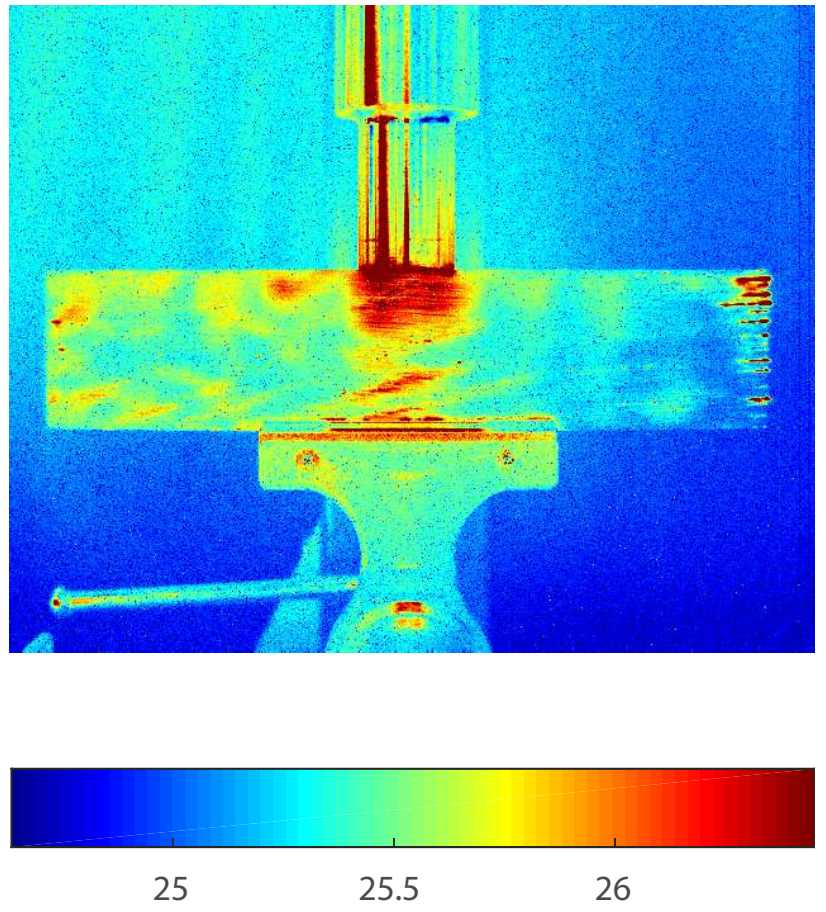

Figure 7: An example lamella in pseudocolor scaled between the 1st and 99th percentile value. The color bar shows the temperature range in degrees Celsius. (Figure best viewed in color)

sequences, it was decided that a good time to extract a raw file of temperature values was at $300 \mathrm{~ms}$ after the beginning of the excitation. At that point the contrast between cracks and solid material was high. The images extracted have $640 \times 512$ pixels and a pixel density of 47 ppi, or 1.85 ppmm.

A lamella with cracks near the left and right edges can be seen in Figure 7. 


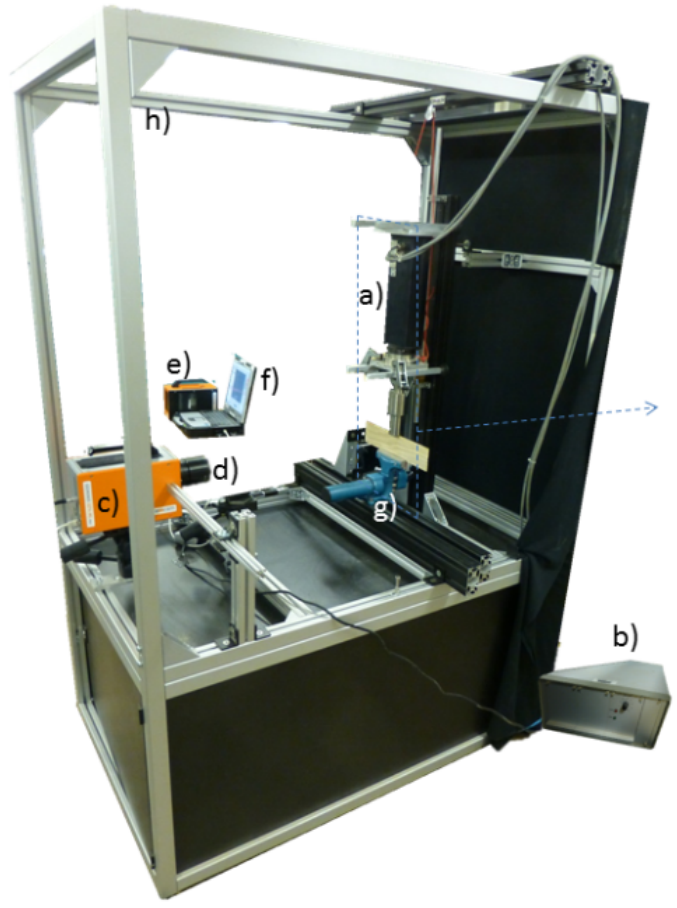

Figure 8: Setup of the ultrasound-excited thermography ${ }_{605}$ experiment. The equipment is listed in Table 2.

\section{Experimental setup}

The equipment used in the UET experiment is listed in Table 2, and Figure 8 shows the experimental setup.

The setup was static in terms of specimen positioning during sequence recording, i.e., all ${ }_{612}$ the specimens assumed the same position in front of an infrared camera. The exact positioning of the lamellae specimens was secured using a vise with a $125 \times 5 \mathrm{~mm}$ groove in it.

The power of the ultrasonic wave was chosen 616 to be $1000 \mathrm{~W}$, which was the maximum power 617 of the ultrasonic generator. During the pre-test ${ }_{618}$ phase, it was concluded that a higher power resulted in a better contrast between the cracks and solid material.

The IR camera was calibrated once every hour during the tests. The calibration was carried out with a black body calibration device by setting the temperature range between $15{ }^{\circ} \mathrm{C}$ and $40{ }^{\circ} \mathrm{C}$.

The distance between the test pieces and the infrared camera lens was set to $60 \mathrm{~cm}$, which was chosen to enclose the whole lamellae with some extra space on the sides.

\section{Pre-processing of images}

The images from the IR camera all include different types of noise and other unwanted effects. Some pre-processing of the IR images is therefore advantageous before the variables are extracted and passed to a classifier. The problems are as follows:

1. Impulse noise over the whole image.

2. Drifting background temperature over time due to temperature rise in the equipment and scene.

3. A minor temperature gradient from left to right and from top to bottom in the images. 
Table 2: A list of the equipment for image acquisition using ultrasound-excited thermography.

\begin{tabular}{lll} 
Label & Name & Type \\
\hline a) & Ultrasonic transducer and a sonotrode & Hielschler ultrasonics $(1000 \mathrm{~W} / 20 \mathrm{kHz})$ \\
b) & Ultrasonic generator & UIP 1000 \\
c) & Infrared camera & IRCAM Geminis $327 \mathrm{k}$ ML pro, $(640 \times 512$ pixels $)$ \\
d) & Lens & MWIR; f/1.5; f = 28mm \\
e) & Temperature calibration device & IRCAM compact blackbody \\
f) & Control unit & Panasonic Toughbook 31 \\
g) & Vise & $/$ \\
h) & Supporting frame & $/$
\end{tabular}

4. Periodic temperature modes originating from the vibration frequency, the shape of the lamellae, and how they were clamped.

5. Very high temperatures of the lamellae regions between the sonotrode and the vise.

Below follows a deeper explanation of each type of noise and the measures taken to mitigate them.

\subsection{Impulse noise}

Impulse noise, also called salt-and-pepper noise, is to some extent always present in the images. The presence of impulse noise can for 635 example be discovered by looking at the his- 636 togram of a background area. Figure 9 shows 637 the histogram of a $30 \times 40$ pixel background ${ }_{638}$ area from the top left corner of Figure 7. The ${ }_{639}$

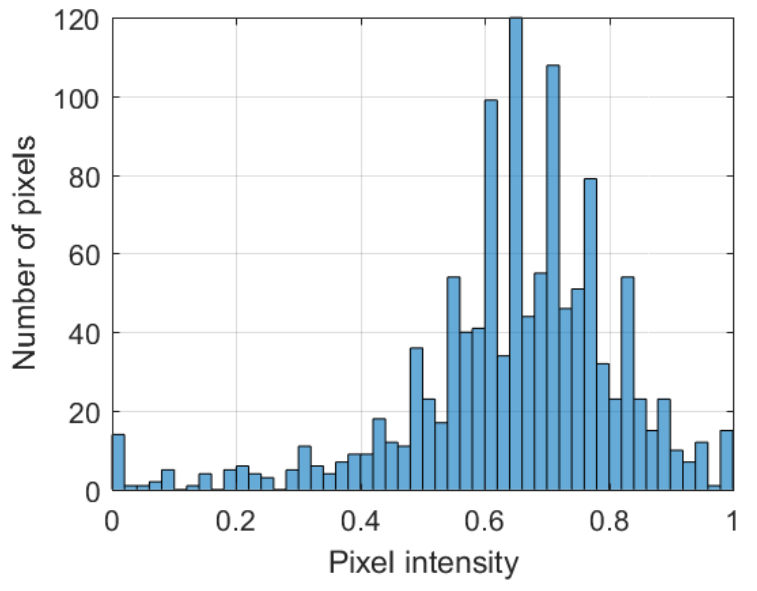

Figure 9: The histogram of a $30 \times 40$ pixel area in the top left corner of Figure 7. The values of the image have been stretched between the 1st and 99th percentile and mapped between 0 and 1 .

spikes at the tail ends of the histogram indicate that there is impulse noise in the image.

Impulse noise can be mitigated by calibrating the temperature range of the infrared camera often, but the noise was also suppressed af- 


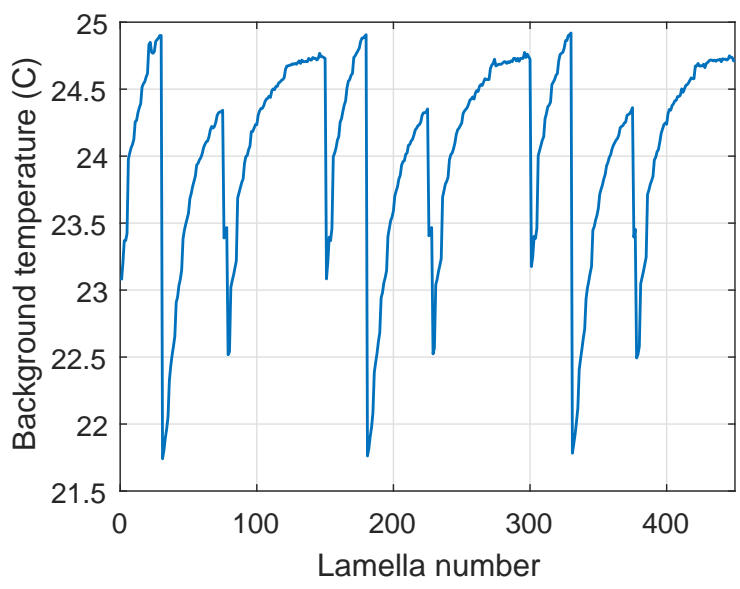

Figure 10: The median temperatures of a $10 \times 10$ pixel background area at the bottom right corner calculated for each lamella image. The lamella ID number is specified on the $x$-axis in the order they were tested. Number ${ }^{665}$ 1-150: no cracks, 151-300: unacceptable cracks, and 666 301-450: acceptable cracks. terwards using a median filter of size $3 \times 3$. The MATLAB function medfilt2 was used with symmetric padding.

\subsection{Suppression of the background tempera- ${ }_{671}$} ture drift

The IR camera was calibrated at certain 673 times during the tests. However, in hindsight, 674 this calibration did not prove to be enough. 675 The median temperatures of a $10 \times 10$ pixel 676 background area at the bottom right corner 677 were calculated for every lamella image (see 678 Figure 10). This area was chosen since it 679 should be the least affected by the rising heat 680 generated from the sonotrode. It is clear that 681 the overall temperature of the IR images is increasing between the points of calibration as the equipment is on. Therefore, this difference in background median value between the first frame and each subsequent frame was calculated and subtracted. Many other areas were also investigated but they all show the same pattern. Hence, it should not matter much which background area is used to subtract the background temperature differences.

According to the room temperature log, the room temperature only varied $\pm 0.2{ }^{\circ} \mathrm{C}$ during the experiments, so subtracting the temporal background change will not introduce a significant error.

\subsection{Scene temperature gradient and tempera- ture modes}

As mentioned, there are slow-changing temperature gradients present in the images, which can be seen in Figure 7. The reason is that the electrical equipment in the setup heats up the scene unevenly, and more so as the equipment is kept on. We want to suppress the gradients over the lamellae to reduce unwanted variability of the input to the classifier.

It was found that the gradients are stronger over the lamellae than in the background, so one cannot simply use the more static back- 
ground to estimate the gradients. There are 710 also periodic temperature modes, hot spots, 711 present over the lamellae which are more or 712 less easy to note depending on the specimen ${ }_{713}$ and the amount of contrast stretching (see Fig- 714 ure 7). The periodic temperature modes can ${ }_{715}$ interfere with the crack detection so we want ${ }_{716}$ to remove them but keep the hot regions where ${ }_{717}$ there are cracks.

The cracks in the images usually appear as ${ }^{719}$ small objects of high temperature either elon- 720 gated in the horizontal direction or as small ${ }^{721}$ disk-shaped spots. Some examples can be seen ${ }^{722}$ close to the left and right edges of the lamella ${ }^{723}$ in Figure 7. These regions make up the infor- ${ }^{724}$ mation that we very much want to keep.

The minor slow-changing gradients and large hot spots can in terms of image processing be treated as uneven lighting and can either be mitigated using a high-pass filter or by estimating the background and subtracting it. The latter approach was chosen due to its simplicity and because it produced more nice-looking images.

In Figure 11, the background has been sub- 734 tracted from the lamella in Figure 7 to pro- 735 duce this final result. The chosen method is 736 explained here in Sections 5.3.1-5.3.2.

\subsubsection{Edge-preserving smoothing}

An edge-preserving smoothing filter was added before the background subtraction to get a cleaner result. This type of filter will smooth out less distinct gradients in the images but will leave the cracks relatively untouched.

The guided filter introduced by $\mathrm{He}$ et al. (2013) was used, which is implemented in the MATLAB function imguidedfilter. This filter behaves like the popular bilateral filter (Tomasi and Manduchi, 1998), but has better behavior near edges, as it avoids gradient reversal artifacts. The neighborhood size was set to $5 \times 5$ and the degree of smoothing to $1 \mathrm{e}-8$ after experimenting with different values.

725 726 727 728 729 730 731 732 


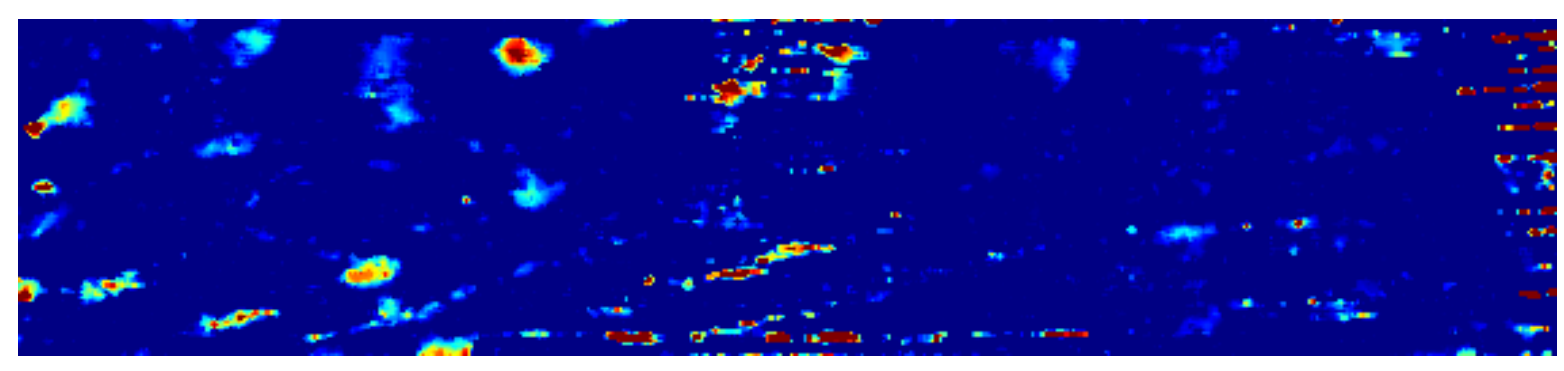

Figure 11: The image resulting after edge-preserving smoothing and gray-scale top-hat by reconstruction applied to the image in Figure 7. (Figure best viewed in color)

stores the larger high intensity regions that re- 759 main after erosion exactly as they were before, 760 through repeated dilation.

In this paper, the opening by reconstruction 762 is performed using the following MATLAB ${ }^{763}$ commands:

mask $=I$

marker $=$ imerode $(I, h)$;

background = imreconstruct(marker, mask);

Matlab's imreconstruct repeatedly dilates the 770 marker image using an 8-connected neighbor- ${ }_{771}$ hood until it fits the border of the mask im- 772 age. The algorithm stops when there are no ${ }_{773}$ more changes between subsequent dilations. 774 The function reconstructs the background us- 775 ing a fast hybrid gray-scale reconstruction al- 776 gorithm described in Vincent (1993).

The flat structuring element, $h$, was chosen 778 in the form of a horizontal line of length 25779 pixels. We assume that cracks are very rarely longer than 25 pixels, which make longer lines or other shapes, e.g., growth rings and large hot spots, become part of the background.

Top-hat by reconstruction means that the background obtained through opening by reconstruction is subtracted from the original image, therefore removing unwanted objects.

For a more thorough explanation of the underlying gray-scale morphology operations, see Gonzalez and Woods (2008, pp. 659-679).

\subsection{Regions of interest}

During manual inspection it was discovered that the cracks mostly appear close to the left and right edges of the lamellae. Therefore, it is most important to gather information there to train a successful classifier. The regions between the vice and sonotrode unfortunately interfere with the classification because the wood gets too hot there (see Figure 7). Hence, these regions were cut away in the 


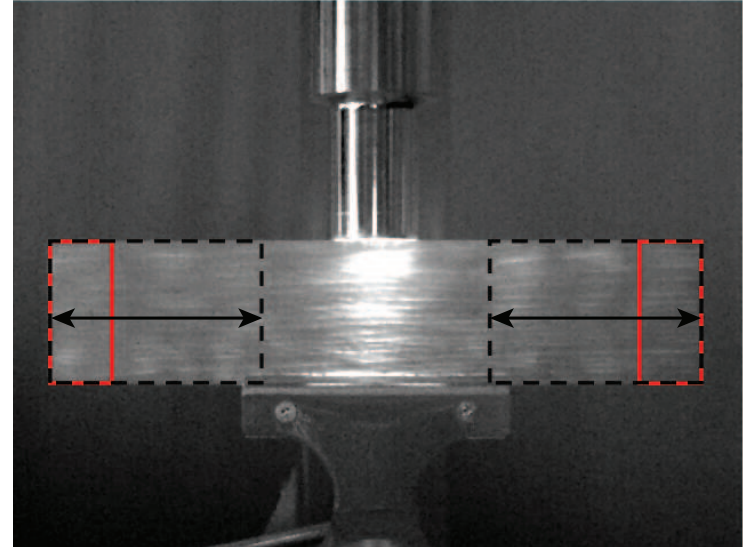

Figure 12: One region from the left side and one re- 803 gion from the right side of the lamella were extracted. 804 The width of these regions was varied, as shown by the black arrows and dashed rectangles. The red solid line rectangles show a width of 55 pixels as reference.

images. The classification accuracy was esti- ${ }_{808}$ mated for varying region widths to find the op- ${ }_{809}$ timal value. The left and right regions of in- ${ }_{810}$ terest and how they were varied is shown in ${ }_{811}$ Figure 12, where the red rectangles mark a re- ${ }_{812}$ gion width of 55 pixels as reference and the black dashed regions show roughly the maxi- ${ }^{813}$ mum width tested.

The classification accuracy was evaluated ${ }_{815}$ for each type of variable, as described in Section 3.3 , while varying the width between $10^{816}$ to 200 pixels with a step length of 5 pixels.

\section{Variable selection and optimal settings}

Searching for good predictor variables, pa- 820 rameter settings, and pre-processing steps, has 821 been a daunting iterative process. The optimal parameter settings change as the preprocessing of the images is changed, and the parameter settings are also affected by adding or discarding predictors. Each pre-processing step, parameter setting, and predictor variable, has been investigated through scientific control, i.e., by keeping all but the parameter in question constant.

Different design choices were investigated by measuring the average 7 -fold CV classification accuracy over 100 randomly partitioned subsets of the cracked lamellae in the training set. Random forests, as implemented in the MATLAB function fitcensemble, were used with 300 learning cycles and all other settings set to the default.

The predictor variables finally chosen were:

1. Maximum temperature.

2. Statistical moments: mean, variance, skewness and kurtosis.

3. Percentiles $92-100$ of the distribution of temperature of the edge-detected pixels in the left and right regions.

4. The Completed LBP histogram with a certain combination of the Sign, Magnitude and Center-operator histograms. 
(Explained in Section 6.3.)

5. Number of foreground pixels after preprocessing and thresholding the regions.

All the above mentioned variables were ${ }^{852}$ standardized after extraction so that they ${ }^{853}$ would initially have an equal impact on the ${ }^{854}$ classification.

According to Hastie et al. (2009), boosted ${ }^{856}$ ensembles and random forests are very robust ${ }^{857}$ towards noisy or collinear variables. There- ${ }^{858}$ fore, no further variable reduction was inves- ${ }^{859}$ tigated until the hyperparameters had been op- ${ }^{860}$ timized.

\subsection{Maximum temperature}

The maximum temperature in a region of in- ${ }^{864}$ terest is often a good predictor of crack pres- ${ }^{865}$ ence on its own. The maximum temperature 866 along with the average temperature is maybe ${ }^{867}$ the most common indicators of faults and de- 868 fects used in the thermography literature.

\subsection{Percentile values of edge pixels}

The histogram, or certain bins of the histogram, could possibly be a good predictor of cracks. However, the temperature distributions are very skew. Most of the pixels are non-cracks and are therefore not heated
48 much. To even out the histograms and discard most of the background pixels, a few methods were evaluated with respect to classification accuracy. Morphological gradient as well as Canny- and Laplacian of Gaussian (LoG) edge detection were used to mask out edges, hopefully near potential cracks.

The MATLAB function edge was used to produce the edge masks. A reasonable threshold interval to investigate for LoG was obtained by calculating the default thresholds for each individual image as returned by edge. The interval to investigate became $1 \mathrm{e}-7$ to 1e-5. Analogously, an interval for $\sigma$ was investigated between 0.5 and 2 .

The histograms obtained after masking are very sparse at the higher temperatures, so percentile values were instead used to represent the distributions. Percentiles are less sensitive to "outliers" and minor shifts in temperature.

The percentile values were calculated separately on the left and right sides.

\subsection{Completed Local Binary Pattern (CLBP)}

The CLBP implementation of Guo et al. (2010) has been used in this study ${ }^{2}$. In addition to the standard signed LBP, explained in

\footnotetext{
${ }^{2}$ http://www. comp.polyu.edu.hk/ cslzhang/code/CLBP. rar, last visited 2016-09-08.
} 


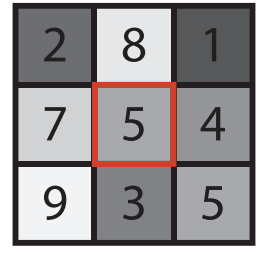

(a)

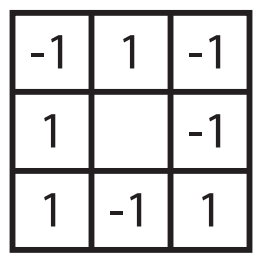

(c)

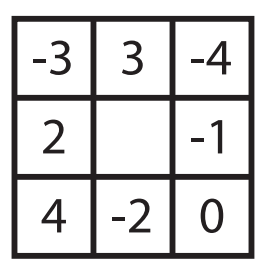

(b)

\begin{tabular}{|l|l|l|}
\hline 3 & 3 & 4 \\
\hline 2 & & 1 \\
\hline 4 & 2 & 0 \\
\hline
\end{tabular}

(d)
Figure 13: (a) An example $3 \times 3$ image block. (b) The ${ }^{898}$ differences between the center pixel and surrounding 899 sample points. (c) The sign differences. (d) The magni- 900 tude components. structed into its corresponding vectors in (c)- 908 (d) using what Guo et al. (2010) refer to as the ${ }_{909}$ Local Difference Sign-Magnitude Transform ${ }_{910}$ (LDSMT), where (c) contains just the signs ${ }_{911}$ and (d) is a descriptor of the magnitude of the ${ }_{912}$ differences.

The magnitude vectors are converted to bi- 914 nary codes by thresholding them by the mean 915 value of all the magnitude values in the im- 916 age. The M-histogram is then built in the same manner as the S-histogram (explained in Section 2.1).

The center pixel binary map comes from thresholding all center pixels by the average gray level of the whole image. The $\mathrm{C}$ histogram is then just the two-component vector, [\# 0,\# 1], of this thresholded image.

There are many ways to pre-process the images before the CLBPs are calculated. Furthermore, there is one region in each side of the lamella, so another question is how to best combine the CLBP histograms. The two histograms were both summed and concatenated to find the best approach. Several CLBP histograms of varying scales and numbers of sample points were also fused together in both concatenated- and joint histograms to assess the importance of different settings.

\subsection{Number of foreground pixels}

Especially the time normalization and background subtraction described in Section 5 are steps that increase the chances to segment cracks successfully. The \# foreground pixels were summed after thresholding the left and right lamellae regions using several threshold levels. The threshold was optimized by measuring the classification accuracy as before. 
The summation of the pixels gives one value 945 for the left region, and one value for the right 946 region. The numbers are measures of how 947 large the supposed crack regions are.

There is no special shape of the hot regions 949 that include cracks. Sometimes the cracks 950 are thin and sometimes they appear circular. 951 It probably depends on the three-dimensional 952 nature of the crack. Therefore, it is not very 953 likely that a shape descriptor would be advan- 954 tageous to the classifier.

\section{Classifiers and hyperparameter opti- mization}

The type of data and its characteristics 959 greatly affect the performance of a classifi- 960 cation method (Kiang, 2003). However, ac- 961 cording to Hamner (2014), ensemble classi- 962 fiers often do well on many different types 963 of data sets. In the last few years, most 964 machine learning competitions held at Kag- 965 gle.com have been won by ensemble classi- 966 fiers. A successful strategy has also typically 967 been to use the out-of-bag (OOB) variable im- 968 portance measure from random forests to find good predictors. Out-of-bag information in- ${ }^{969}$ cludes observations and predictors that were 970 not used in the training of a particular tree clas- 971 sifier. The OOB variable importance in MAT- 972
LAB is calculated by estimating the out-of-bag classification error while permuting the predictors for each tree. If the mean difference of the error is high for a predictor, then it is seen as an important variable.

MATLAB's Classification Learner application was used to quickly survey many classification methods, such as discriminant analysis, support vector machines, nearest neighbor, logistic regression, and ensemble classifiers. It became clear that the ensemble methods: boosted trees and random forests, performed the best using default settings. Hence, ensemble methods were chosen for a more thorough evaluation.

As already mentioned, the accuracy of the ensembles was investigated using 7-fold crossvalidation on 100 randomly partitioned subsets of the cracked lamellae in the training set. When evaluating the performance of the ensembles on the external test set, 1000 randomly partitioned subsets of the cracked lamellae were used to obtain even more correct statistics.

\subsection{Hyperparameter optimization}

MATLAB was set to automatically optimize all available hyperparameters for the classification ensembles. The default variable ranges 
were left unchanged but the number of ob- 1000 jective function evaluations was increased to 1001 1000. The default method, Bayesian optimiza- 1002 tion, was used to search for optimal hyperpa-1003 rameters. The 7-fold loss with classification ${ }_{1004}$ error specified as the loss function was used ${ }_{1005}$ as the performance metric. All available hy-1006 perparameters and their options are stated in ${ }_{1007}$ Table 3.

The time-consuming hyperparameter opti- 1009 mization was run ten times, meaning ten differ- 1010 ent instances of undersampling of the lamellae 1011 group including cracks.

It is difficult to find only one optimal classi- 1013 fier in this way since the data is always chang- 1014 ing, and it is not reasonable to calculate an ${ }_{1015}$ average of the hyperparameters. But the ob-1016 tained classifier settings should probably per-1017 form quite well on similar data.

\section{Results and discussion}

In this section, the general findings and set- ${ }^{1020}$ tings are first presented for each variable type. ${ }^{1021}$ Then, the performance of the ensembles are ${ }^{1022}$ explained and discussed.

\subsection{Left and right lamellae regions}

During evaluation of the CLBP histograms 1026 it was discovered that the left and right regions 1027 do not provide an equal amount of information about eventual cracks. This odd behavior was also confirmed for all the other variable types, and it was thereafter verified by carefully looking at the images and trying to detect cracks manually. The fact was that the left sides more often aided a correct classification than the right sides. True cracks were easier to spot on the left sides. Our best guess is that the vice did not clamp the lamellae exactly equally on the left and right sides at all times. The problem should not lie in the material, because the lamella face that was facing the camera was chosen at random.

The classification accuracy was the highest for all variable types when information was gathered from the left and right regions separately.

\subsection{Chosen pre-processing and parameter} settings

It was found that different region widths and pre-processing methods were optimal for different types of variables. The optimal widths for each type of variable and their classification accuracy are stated in Table 4. According to this table, the CLBP histogram and \# foreground pixels are the most important variable types, but only by a small margin. 
Table 3: List of hyperparameters to optimize, their data type, range and transform.

\begin{tabular}{r|l} 
Method & Categorical: $\{$ Bag, GentleBoost, LogitBoost, AdaBoost, RUSBoost $\}$ \\
NumLearningCycles & Integer: [10,500], Transform: log \\
LearnRate & Integer: [0.001, 1], Transform: log \\
MinLeafSize & Integer: [1,83], Transform: log \\
MaxNumSplits & Integer: [1, 165], Transform: log \\
SplitCriterion & Categorical: \{Gini’s diversity index, deviance (cross entropy)\} \\
Num VariablesToSample & Integer: [1, all], Transform: none
\end{tabular}

Table 4: Optimal region widths (in pixels) with respect to cross-validated classification accuracy on the training data for different variable types.

\begin{tabular}{l|r|r} 
Variables & Optim. width $(\mathbf{p x})$ & Mean class. acc. $(\%)$ \\
\hline Maximum temperature & 70 & $77.0(\mathrm{SD}=3.6)$ \\
Statistical moments & 20 & $78.2(\mathrm{SD}=3.3)$ \\
Percentile values & 80 & $79.2(\mathrm{SD}=3.0)$ \\
CLBP histogram & 25 & $\mathbf{8 1 . 4}(\mathrm{SD}=2.5)$ \\
\# foreground pixels & 135 & $\mathbf{8 1 . 1}(\mathrm{SD}=2.3)$
\end{tabular}

All predictors benefited from the inclu-1039 sion of edge-preserving smoothing in the pre-1040 processing. However, only \# foreground pix-1041 els and percentile values benefited from having the background subtracted using opening by reconstruction. Therefore, it was only applied for those variables.

\subsubsection{Percentile values of edge pixels}

Laplacian of Gaussian (LoG) edge detection 1045 was chosen since it produced the most uniform 1046 histograms out of the three alternatives. $\operatorname{LoG}_{1047}$ was used with $\sigma=1$ and filter size $7 \times 7$. A fixed threshold, 1e-6, was chosen because it gave a high classification accuracy. most important when the accuracy was evaluated for different percentile combinations. The OOB variable importance also encouraged the choice. 


\subsubsection{CLBP histograms}

The CLBP texture descriptor worked best when using narrow image regions, as seen in Table 4. It apparently captures most of its cracks close to the edges. Unfortunately, the right sides of the lamellae only worsened the classification accuracy. They were therefore omitted as input to the final classifier for the CLBP variables. Thus, eventual cracks on the right sides had to be captured by the other variable types.

The best results were obtained using eight sample points and a radius of one pixel. When 1084 increasing the number of sample points and 1085 adding more scales (more radii) to the $\mathrm{CLBP}_{1086}$ histogram the results only worsened.

Rotation invariant uniform patterns were ${ }_{1088}$ chosen since these led to higher classification ${ }_{1089}$ accuracy than any combination that included ${ }_{1090}$ either upright or non-uniform patterns.

The best found combination of the three 1092 CLBP histograms was:

$\left[C L B P \_S, C L B P \_M, C L B P \_S \bullet C L B P \_M, \ldots\right.$

\section{CLBP_C],}

where $\bullet$ denotes elementwise multiplication. 1098 Creating 2D or 3D histograms and joining ${ }_{1099}$ them as described by Guo et al. (2010) did not ${ }_{1100}$

improve the classification accuracy.

\subsubsection{Number of foreground pixels}

According to Table 4, the \# foreground pixels variable uses information from a larger part of the lamellae.

A good combination of region width and threshold level was found by estimating the classification accuracy while alternately varying the region width and applying different threshold levels. The threshold was fine tuned to 0.0022 .

\subsection{Individual variable importance}

The top ten individually most important variables are shown in Figure 14. The order of the important variables changes only slightly if the number of learners is reduced. Apparently the variables extracted from the left regions contribute more than those from the right, as all of the top ten variables are from the left.

Most of the important variables in some way describe the temperature signature at the high end of the heat distribution. The kurtosis on the left side, and to some extent also the skew- 


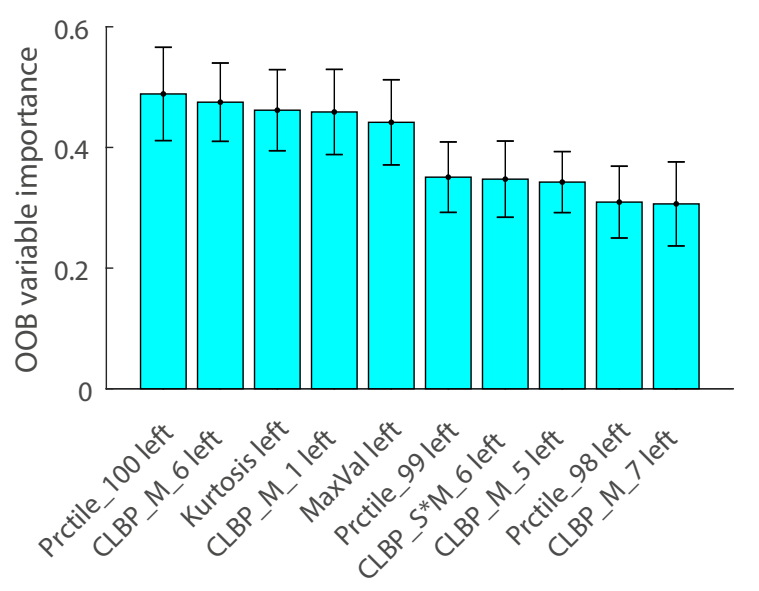

Figure 14: Top ten important variables according to the ${ }_{1123}$ mean OOB variable importance averaged from 100 random samplings of the training data using random forests with 300 learners. The error bars extend to \pm 1 standard $^{1125}$ deviation.

remaining statistical moments on both left and right side are not very important.

The percentile values $98-100$ on the left ${ }_{1131}$ side are ranked as important, but most of the 1132 other percentile values are located in the mid- 1133 dle, i.e., not very important.

The CLBP histograms are not trivial to interpret, but the frequency and magnitude of edge/line/corner-like features (CLBP_M_5-7) and spots (CLBP_M_1) are important.

The two variables containing the number of ${ }_{1140}$ foreground pixels ended up just outside this ${ }_{1141}$ ranking list, in places 11 and 14.

\subsection{Classifiers and hyperparameters}

As mentioned earlier, the hyperparameter optimization was given full freedom to optimize all applicable settings for the ensemble methods. Nevertheless, when the "optimized" ensembles were later evaluated on the training data, they all got slightly worse accuracies than the original RF classifier. The original RF obtained a mean classification accuracy of $0.82(\mathrm{SD}=0.025)$ on the training data, while the optimized ensembles were all located close to 0.8. However, the optimized ensembles achieved comparably higher mean accuracies when run on the external test set, see Figure 15. On the external test set, most optimized ensembles perform better than the original RF and make a mistake about every fifth lamella.

This result is similar to what we got ourselves when classifying all images manually (0.83).

It is likely some minor overfitting occurred on the relatively small training set, both in variable selection and in model selection (Cawley and Talbot, 2010).

The area under the receiver operating characteristic (ROC) curve was also calculated as a measure of how the ensembles would perform more generally, independently of the operating point. Figure 16 shows that the areas under the curves (AUC) are fairly high for all these en- 


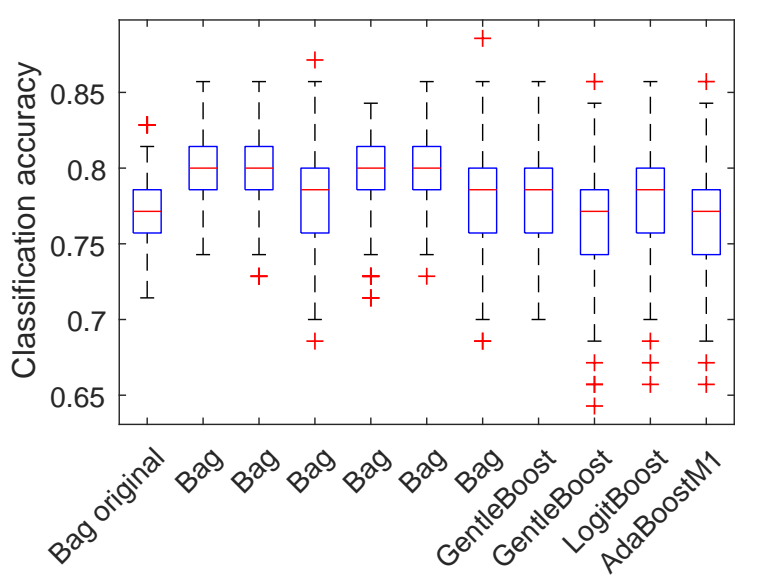

Figure 15: The classification accuracy for the original and the ten optimized classification ensembles on the external test set. The statistics were calculated using 1000 ensembles and undersamplings. Whiskers extend to \pm 2.7 standard deviations.

sembles, which means that it is indeed possible to get a fairly good separation between the two 1158 classes.

A confusion matrix was calculated for the ${ }^{1160}$ third ensemble method in Figures $15-16$ by ${ }^{1161}$ summing the outcomes from the 1000 repeti- ${ }^{1162}$ tions, see Figure 17. The third method was ${ }^{1163}$ chosen as an example because it had a top ${ }^{1164}$ score in both accuracy and AUC, but the con- ${ }^{1165}$ fusion matrices of all tested methods were ${ }^{1166}$ quite similar $\left(\mathrm{TN} \approx 43 \%-45 \%, \mathrm{FN} \approx 13 \%-{ }_{1167}\right.$ $17 \%, \mathrm{FP} \approx 5 \%-7 \%, \mathrm{TP} \approx 33 \%-37 \%)$. The op- 1168 timal operating points of all methods tended to 1169 slightly prioritize guessing the no-crack class 1170 and thus they misclassify more of the lamellae ${ }_{1171}$

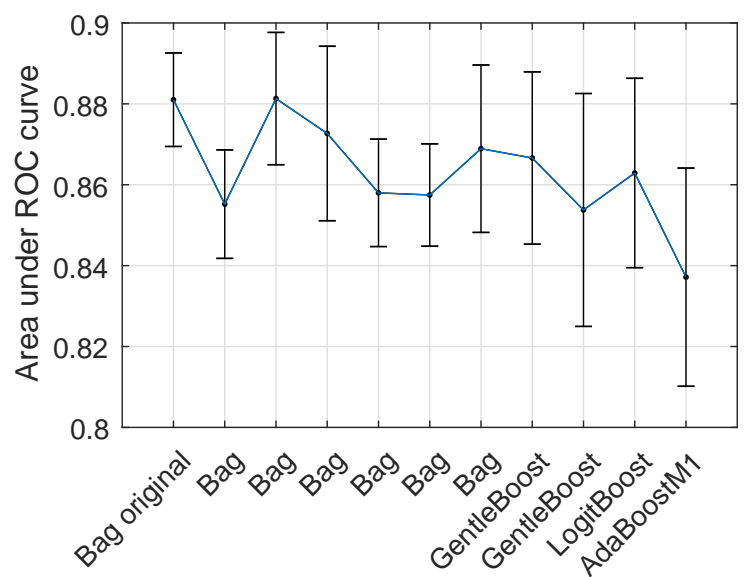

Figure 16: The AUC for the original and the ten optimized classification ensembles on the external test set. $\mathrm{Bag}$, or bootstrap aggregation, is only a more general term for a random forest. The statistics were calculated using 1000 ensembles and undersamplings. The whiskers extend to \pm 1 standard deviation.

with cracks. However, the operating point can of course be tuned according to preference, at the cost of lowering the total accuracy slightly. In our case, where the test set is balanced and the cost for misclassification was set equally for both classes, MATLAB essentially picks the operating point by finding the vector index that maximizes True Positive Rate - False Positive Rate.

Most optimized ensembles performed quite similarly on the test set, and as a random forest was found optimal 6/10 times and a boosting method 4/10 times, it is difficult to declare a winning method. The random forests were 


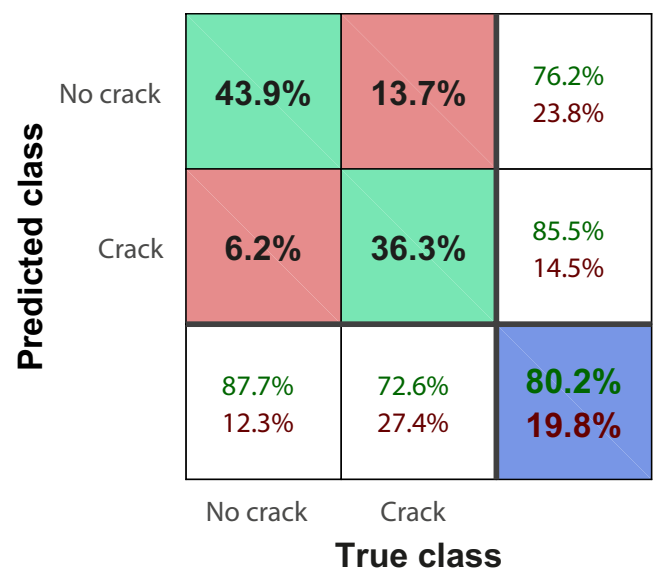

Figure 17: The summed confusion matrix of method $_{1195}$ number three in Figures 15-16 from 1000 repetitions of trained ensembles and undersamplings.

all built with 10-14 number of learning cycles (decision trees), otherwise there were no distinct patterns as to which hyperparameters were successful.

If the pre-processing is removed altogether, ${ }_{1203}$ all tested ensembles get average accuracies be- ${ }_{1204}$ tween $0.71-0.74$ on the external test set.

When calculating the mean classification ${ }_{1206}$ accuracy on the test set with increasing num- 1207 ber of important variables, it was noted that af- 1208 ter about 25 variables, the accuracy started to 1209 decline somewhat. This indicates that the rest ${ }_{1210}$ of the variables do not contribute to the perfor- 1211 mance. Reducing the number of variables to 1212 the 25 most important did not produce any sig- 1213 nificantly different results on either training- or 1214 external test-set. Using only one of the topmost important variables gives a classification accuracy of about 0.75 on the test set. Adding more variables, however, will help boost the performance those few extra valuable percentage points.

\subsection{Analysis of results}

The accuracies of the classifiers can be seen as quite high, taking into account that the classifiers performed similarly to our own attempt at manually separating the classes. This outcome also suggests that there might not be information present in the images to get much higher accuracy. Furthermore, although the lamellae were investigated via microscope, there is still the possibility that a few lamellae actually should have been assigned to the opposite class.

The classification accuracy obtained in this paper is a significant improvement over the previous results by Popovic (2015), where a classification accuracy of only 0.6 was obtained. Nevertheless, it is not fruitful to make a meticulous comparison with the previous results, since the null accuracy, i.e., the accuracy obtained by always guessing the majority class, then was $332 / 450=0.74$. 


\section{Summary and Conclusions}

We studied the possibility of using ensem- 1244 ble methods: random forests and boosting, to 1245 detect cracks using vibrothermography and a 1246 variety of predictors. When friction occurs in ${ }_{1247}$ thin cracks, they become warm and thus visi- 1248 ble to a thermographic camera.

The classification accuracy was signifi-1250 cantly improved from the previous work by ${ }_{125}$ Popovic (2015) through added image process- 1252 ing, the introduction of more predictors, and ${ }_{1253}$ the use of automated machine learning. Sev-1254 eral image processing techniques were used ${ }_{1255}$ to suppress the noise and enhance possible ${ }_{1256}$ cracks, reducing the variance between the im-1257 ages.

Most variables were used to capture the 1259 upper part of the heat distribution, such as 1260 the maximum temperature, kurtosis, and per- 1261 centile values $92-100$ of the edge pixels in the ${ }_{1262}$ image. Texture in the images was captured by ${ }_{1263}$ Completed LBP histograms and we also seg-1264 mented cracks through background suppres- 1265 sion and thresholding.

The best ensembles reach an average classi- 1267 fication accuracy close to 0.8 , which is a $\operatorname{good}_{1268}$ result and similar to our own manual attempt ${ }_{1269}$ at separating the images $(0.83)$.

\section{Future work}

This study revealed an unfortunate flaw in the experimental setup, where it is likely that the vise did not clamp the lamellae perfectly equally on each side. In future research this possible flaw should be corrected or maybe even some alternative methods of clamping could be investigated.

It would be interesting to try a variable frequency ultrasonic source to be able to maximize the SNR between cracks and solid material. A possibility would be to sweep through many frequencies and combine the images to suppress the heat addition from standing waves (Guo and Vavilov, 2013). Even so, in our case there was only a small temperature difference between the highs and lows of the nodal points.

Another enhancement could be to try to incorporate the shape of the salient features that appear in the IR images. If the shapes of the features are analyzed, maybe some false cracks can be discarded as being something else.

A better result could probably be found by optimizing the duration of excitation before the snapshot was taken, as this was only chosen by naked eye. 
Typically, the sign histogram of the CLBP ${ }_{1299}$ carries most of the information according to 1300 Guo et al. (2010). However, in our case, the ${ }_{1301}$ magnitude histogram seems to contribute more if we look at the OOB variable importance. ${ }_{1302}$ This is something that could be investigated further.

There are many ways to handle imbalanced ${ }^{1304}$ data sets. An interesting approach to try is to ${ }_{1306}$ combine undersampling of the majority class 1307 and oversampling of the minority class as was ${ }^{1308}$ done by Liu et al. (2006).

There are many classification methods ${ }_{1311}$ available which could be evaluated in future ${ }_{1312}$ work. For example, SVMs showed quite good 1313 results after reducing the number of input vari- ${ }^{1314}$ ables. It is possible that a dimensionality re- ${ }^{1315}$ 1316 duction technique such as Principal Compo- ${ }_{1317}$ nent Analysis (PCA) could also have a positive ${ }_{1318}$ effect on the results.

\section{Acknowledgments}

1321

The authors would like to thank Tarkett ${ }_{1323}$ for providing us with the material and for ${ }_{1324}$ lending us their resources. Thanks to Peter ${ }^{1325}$ Meinlschmidt and Jochen Aderhold at Fraun- ${ }^{1326}$ WKI for making the UET testing possi- ${ }^{1327}$ hofer WKI for making the UET testing possi- ${ }_{1328}$ ble and for answering our questions and be- ${ }_{1329}$ ing helpful in many ways. We would also ${ }_{1330}$ like to thank Anders Landström, LTU, for a good initial discussion and help regarding preprocessing of the raw images.

\section{References}

Aggelis, D., Kordatos, E., Soulioti, D., Matikas, T., 2010. Combined use of thermography and ultrasound for the characterization of subsurface cracks in concrete. Construction and Building Materials 24, $1888-1897$.

Ahonen, T., Member, S., Hadid, A., Pietikinen, M., Member, S., 2006. Face description with local binary patterns: Application to face recognition. IEEE Transactions on Pattern Analysis and Machine Intelligence 28, 2037-2041.

Bagavathiappan, S., Lahiri, B., Saravanan, T., Philip, J., Jayakumar, T., 2013. Infrared thermography for condition monitoring a review. Infrared Physics \& Technology 60, $35-55$.

Berglind, H., 2003. Non-destructive detection of glue deficiency in laminated wood using thermography. Ph.D. thesis. URL: http: //ltu.diva-portal.org/smash/get/diva2: 990194/FULLTEXT01 .pdf. Accessed May 5, 2017.

Bergstra, J., Bengio, Y., 2012. Random search for hyper-parameter optimization. J. Mach. Learn. Res. 13, 281-305.

Bergstra, J., Yamins, D., Cox, D.D., 2013. Making a science of model search: Hyperparameter optimization in hundreds of dimensions for vision architectures. ICML (1) 28, 115-123.

Breiman, L., 2001. Random forests. Machine learning $45,5-32$. 
Breiman, L., Friedman, J., Olshen, R., Stone, C., 1984. 1365 Classification and Regression Trees. Wadsworth and 1366 Brooks, Monterey, CA.

Brownlee, J., 2015. 8 Tactics to Combat Imbal-1368 anced Classes in Your Machine Learning Dataset. 1369 URL: http://machinelearningmastery . com/. 1370 Accessed Mars 24, 2017.

Cawley, G.C., Talbot, N.L., 2010. On over-fitting in 1372 model selection and subsequent selection bias in per- 1373 formance evaluation. J. Mach. Learn. Res. 11, 2079-1374 2107.

1375

Eriksson, L., Johansson, E., Kettaneh-Wold, N., Wold, 1376 S., . Multi- and megavariate data analysis: Basic 1377 principles and applications. v. 1.

Freund, Y., Schapire, R.E., 1997. A decision-theoretic 1379 generalization of on-line learning and an application 1380 to boosting. J. Comput. Syst. Sci. 55, 119-139. 1381

Freund, Y., Schapire, R.E., 1999. A short introduction 1382 to boosting, in: In Proceedings of the Sixteenth Inter- 1383 national Joint Conference on Artificial Intelligence, 1384 Morgan Kaufmann. pp. 1401-1406.

Friedman, J., Hastie, T., Tibshirani, R., 1998. Additive 1386 logistic regression: a statistical view of boosting. An- 1387 nals of Statistics 28, 2000.

1388

Global Industry Analysts, Inc., 2017. Wood flooring - a 1389 global strategic business report. Accessed September 1390 $14,2017$.

1391

Gonzalez, R., Woods, R., 2008. Digital Image Process- 1392 ing (3rd Edition). Pearson Prentice Hall.

1393

Guo, X., Vavilov, V., 2013. Crack detection in alu- 1394 minum parts by using ultrasound-excited infrared 1395 thermography. Infrared Physics and Technology 61, 1396 149-156.

1397

Guo, Z., Zhang, L., Zhang, D., 2010. A completed mod- 1398 eling of local binary pattern operator for texture classification. Trans. Img. Proc. 19, 1657-1663.

Hamner, B., 2014. Yet another Conference 2014. Machine learning best practices we've learned from hundreds of competitions. URL: https://youtu. be/9Zag7uhjdYo. Accessed Feb. 27, 2017.

Hastie, T., Tibshirani, R., Friedman, J., 2009. The Elements of Statistical Learning: Data Mining, Inference, and Prediction, Second Edition. Springer Series in Statistics, Springer New York.

He, K., Sun, J., Tang, X., 2013. Guided image filtering. IEEE Transactions on Pattern Analysis and Machine Intelligence 35, 1397-1409.

Hutter, F., Hoos, H.H., Leyton-Brown, K., 2011. Sequential Model-Based Optimization for General Algorithm Configuration. Springer Berlin Heidelberg, Berlin, Heidelberg. pp. 507-523.

Infographic Journal, 2013. The Rise of Engineered Wood Flooring by Irma Wallace. Accessed September 14, 2017.

Kiang, M.Y., 2003. A comparative assessment of classification methods. Decision Support Systems 35, $441-454$.

Lamb, F.M., 1992. Splits and cracks in wood, in: Proceedings of the 43rd Annual Meeting of Western Dry Kiln Association, Reno, NV. USA May 13-15, pp. $16-24$.

Liu, Y., An, A., Huang, X., 2006. Boosting Prediction Accuracy on Imbalanced Datasets with SVM Ensembles. Springer Berlin Heidelberg, Berlin, Heidelberg. pp. 107-118.

López, G., Basterra, L.A., Ramón-Cueto, G., de Diego, A., 2014. Detection of singularities and subsurface defects in wood by infrared thermography. Interna- 
tional Journal of Architectural Heritage 8, 517-536. 1433

Lukowsky, D. Meinlschmidt, P.G.W.., 2008. Ultra-1434 schallangeregte thermographie an holzverklebungen 1435 - entwicklung einer prfmethode. Holztechnologie 1436 Holztechnologie 49, No.5, Dresden, Germany, 42-1437 47.

Maldague, X., 2001. Theory and practice of infrared 1439 technology for nondestructive testing. Wiley series 1440 in microwave and optical engineering, Wiley.

1441

MathWorks MATLAB, 2016. Statistics and Machine 1442 Learning Toolbox: 2016a - Ensemble Methods, 1443 The MathWorks Inc., Natick, Massachusetts, United 1444 States.

Meinlschmidt, P., 2005. Thermographic detection of de- 1446 fects in wood and wood-based materials, in: 14th in-1447 ternational Symposium of non-destructive testing of 1448 wood. Hannover, Germany.

Ojala, T., Pietikäinen, M., Mäenpää, T., 2002. Mul- 1450 tiresolution gray-scale and rotation invariant texture 1451 classification with local binary patterns. IEEE Trans. 1452 Pattern Anal. Mach. Intell. 24, 971-987.

Ojala, T., Pietikinen, M., 1999. Unsupervised tex-1454 ture segmentation using feature distributions. Pattern 1455 Recognition 32, 477 - 486 .

Pham, V., 2016. Bayesian optimization for hyper-1457 parameter tuning. URL: https://arimo.com/ 1458 data-science/2016/. Accessed Jan. 23, 2017.

Popovic, D., 2015. Crack Detection and Classification 1460 of Oak Lamellas using On-Line and Ultrasound Ex-1461 cited Thermography. Master's thesis. Luleå Univer- 1462 sity of Technology. Department of Engineering Sci- 1463 ences and Mathematics. Division of Wood Science 1464 and Engineering, Skellefteå.

Rasmussen, C.E., Williams, C.K.I., 2006. Gaussian 1466
Processes for Machine Learning. The MIT Press.

Schapire, R.E., 2013. Explaining AdaBoost. Springer

Berlin Heidelberg, Berlin, Heidelberg. pp. 37-52.

Sembach, J. Wu, D.S.A.H.G., Busse, G., 1997. Nondestructive evaluation of delaminations in laminated wood-based panels by thermographical inspection tools, in: Proc. of workshop on nondestructive testing of panel products. 11 October, Llandudno, UK, J. Hague. pp. 41-48.

Shen, H.B., Yang, J., Chou, K.C., 2007. Euk-ploc: an ensemble classifier for large-scale eukaryotic protein subcellular location prediction. Amino acids 33, 5767.

Snoek, J., Larochelle, H., Adams, R.P., 2012. Practical bayesian optimization of machine learning algorithms, in: Advances in neural information processing systems, pp. 2951-2959.

Thornton, C., Hutter, F., Hoos, H.H., Leyton-Brown, K., 2013. Auto-weka: Combined selection and hyperparameter optimization of classification algorithms, in: Proceedings of the 19th ACM SIGKDD international conference on Knowledge discovery and data mining, ACM. pp. 847-855.

Tomasi, C., Manduchi, R., 1998. Bilateral filtering for gray and color images, in: Computer Vision, 1998. Sixth International Conference on, IEEE. pp. 839846.

Tsamardinos, I., Rakhshani, A., Lagani, V., 2014. Performance-Estimation Properties of CrossValidation-Based Protocols with Simultaneous Hyper-Parameter Optimization. Springer International Publishing, Cham. pp. 1-14.

Vincent, L., 1993. Morphological grayscale reconstruction in image analysis: Applications and efficient al- 
gorithms. IEEE transactions on image processing 2 , 176-201.

Wagner, P., 2011. Local Binary Patterns. URL: https://bytefish.de/blog/local_binary_ patterns/. Accessed June 15, 2016.

Weiss, S.M., Indurkhya, N., 1994. Decision tree pruning: biased or optimal?, in: AAAI, pp. 626-632.

Wu, D., Busse, G.., 1995. Remote inspection of wood with lock-in-thermography, in: TAPPI 1995 European plastic laminates symposium. Vol 79, N 8. pp. 119-123.

Zhang, H., Zhao, D., 2004. Spatial histogram features for face detection in color images, Advances in Multimedia Information Processing - PCM 2004: 5th Pacific Rim Conference on Multimedia, Tokyo, Japan, November 30 - December 3, 2004. Proceedings, Part I, 377-384.

Zhao, G., Pietikainen, M., 2007. Dynamic texture recognition using local binary patterns with an application to facial expressions. IEEE Trans. Pattern Anal. Mach. Intell. 29, 915-928. 